\title{
EVALUACION DE RESIDUOS DE ASERRIO
}

\author{
Leticia Guevara Salnicov* \\ Pedro Reyes Inca ** \\ Luis Bocanegra Dávila ***
}

\section{RESUMEN}

Dentro del Subproyecto "Adecuación de Tecnología para la Utilización de Residuos" y para proponer usos a los residuos de aserrío se hizo una evaluación cuantitativa y cualitativa. Se efectuó una caracterización de las industrias de Pucallpa, mediante una encuesta realizada en 21 aserraderos.

Luego se evalué residuos de aserrío de ocho maderas: catahua, cedro, caoba, copaiba, cumala, ishpingo, moena y tornillo. El volumen de aserrín se determiné en función del espesor de la sierra y área de corte. Para residuos sólidos se identificó previamente formas frecuentes y características de cada tipo de residuos (cantoneras, cantos y puntas); luego en laboratorio, con réplicas a escala y mediante el método de determinación del volumen aparente por desplazamiento en agua se estableció factores de forma que se los aplicó al cálculo del volumen de residuos, a partir del máximo valor medido del espesor, ancho y longitud. Los principales residuos de aserrio de ocho maderas tienen los siguientes valores promedio: cantos 10,7; puntas 10,2; cantoneras 6,7 ; y aserrín $8,2 \%$. El rendimiento calculado como la diferencia entre el volumen de madera rolliza ingresada y el volumen de residuos de aserrín, expresado en porcentaje es en promedio de $549 \%$ con un valor mínimo de 42,8 para cumala y un máximo de $61,5 \%$ para catahua.

De acuerdo a las dimensiones y factores de forma frecuentes, se propone el uso de puntas para producción de "tejas" y "ladrillos" de madera y aserrín para tableros aglomerados o mineralizados y briquetas.

\section{INTRODUCCION}

Dentro de la transformación mecánica de la madera, la industria del aserrío alcanza alrededor del $90 \%$ del volumen procesado. Esta industria es la más importante y la que más atención ha tenido en estudios de problemática y alternativas de desarrollo. En todos los estudios se concluye que uno de los principales problemas, el costo de la madera aserrada, es causado principalmente por el deficiente abastecimiento de materia prima, por la inefectiva utilización de la capacidad instalada y por la ausencia de tecnología adecuada, especialmente en el reciclado de productos secundarios.

Se estima un valor de $52 \%$ corno rendimiento promedio de aserrío en Pucallpa. Es probable que en un estudio exhaustivo se determine rendimientos

\footnotetext{
* Ing ${ }^{0}$ Forestal. Investigador IIAP

** M.Sc. Forestal. Investigador IIAP

*** Ing $^{\circ}$ Forestal. Investigador IIAP
} 
individuales para cada tipo de madera, en función de la calidad de troza y defectos que in e en el rendimiento y que varían entre maderas.

En 1991 se procesó alrededor de $225000 \mathrm{~m}^{3}$ de madera rolliza, que generan aproximadamente $106000 \mathrm{~m}^{3}$ de residuos, entre cantoneras? cantos, puntas y aserrín. Generalmente las cantoneras se las dimensiona y comercializa para uso como linderos; con los cantos se fabrica palos de escobas, listones, parquet y carbón. Las puntas se las vende como leña o junto con el aserrín son quemados o arrojados a los ríos, provocando costos adicionales de limpieza, transporte y contaminación ambiental.

El uso racional del recurso forestal en plantas de transformación primaria debe traer consigo en una reducción del volumen de residuos. Para esto se requiere una evaluación cualitativa y cuantitativa de residuos a fin de proponer alternativas de uso y el desarrollo de tecnologías de uso de residuos que no requieran mayor inversión.

Este estudio tiene como objetivo propiciar el uso integral y sostenido de la madera y proponer tecnologías para el máximo aprovechamiento produciendo utilidades adicionales, puestos de trabajos y reduciendo la contaminación ambiental. Se plantea las siguientes metas:

1. Evaluación cuantitativa de los residuos de aserrío.

2. Producción de "tejas" y "ladrillos" de madera a partir de residuos de aserrio.

\section{REVISION DE LITERATURA}

Aun cuando la industria de transformación mecánica de la madera es relativamente antigua en nuestro país, todavía no ha podido superar deficiencias. Según Cornejo (1991), los problemas de la actividad maderera pueden aL"ruparse en cuatro categorías: a) accesibilidad, disponibilidad y calidad de los recursos usados en la producción (básicamente madera rolliza); b) baja eficiencia de uso de los recursos actualmente disponibles; c) marco socio-político legal en el que se desenvuelve la producción; y d) los referentes al mercado y normalización de productos terminados (principalmente ausencia de canales formales y constantes de comercialización y carencia de normas técnicas). En la categoría b) se encuentran los problemas referentes a las tecnologías usadas y su adecuación a la realidad económica, social y cultural de la Región, por ejemplo, eficiencia en el uso de la materia prima, impacto ecológico dé las operaciones técnicas de reducción de costos y costo de creación de puestos de trabajo. Según FAO (1980), los problemas de la industria de aserrío en orden de importancia son: abastecimiento de materia prima, ubicación de plantas industriales, maquinaria y equipo de recuperación, organización, distribución y control, normalización, iniciativa empresarial y capacitación. 
La Memoria Anual de la Dirección Regional Forestal y de Fauna (1991) indica que ocho especies maderables cubren el 92,6 \% de la producción de Pucallpa: tornillo (37.1\%); catahua (17.2\%); cedro (10.8\%); caoba (7.3\%); cumala $(6.8 \%)$; copaiba $(6.5 \%)$; moena $(3.9 \%)$ e ishpingo $(2.6 \%>$. Con respecto a los diámetros mínimos de corte para aserrío, Tenazoa (1992) indica los siguientes valores: caoba, 0,64 m; catahua, 0,60 m; cedro de altura, 0,56 m; ishpingo, 0,56 m; moena 0,46 m; y tomillo, 0,61 m. En un estudio de 38 aserraderos de Pucallpa, Gauthier (1986) encuentra un rendimiento promedio de 53,1\% referido al diámetro promedio de las trozas. En un estudio sobre ubicación industrial, Fullop y Vásquez (1989) indican que puede asumirse $54 \%$ como rendimiento promedio de aserrío, quedando $46 \%$ de residuos, de los que, de acuerdo a la clase de madera, puede reciclarse en volumen variable, quedando un residuo no aprovechable constituido en su mayor parte por aserrín. Otero (1985) indica que la industria de transformación mecánica produce importantes volúmenes de residuos, con dimensiones y formas inferiores a las mínimas toleradas como productos comerciales. Otros residuos tienen características tísico-mecánicas diferentes a la madera. Las porciones de madera sólida provienen de secciones de las trozas con forma redondeada (cantoneras, largueros y costaneras); de las secciones con defectos y formaciones no toleradas en los productos terminados; y de los extremos irregulares de la troza. Esta proporción es muy variable y puede ser calculada a partir de muestreos, los cuales dependen del nivel tecnológico e a industria, de las dimensiones de los productos comerciales terminados y sus tolerancias, y de las dimensiones y características de las trozas. Quinteros (1981) opina que la calidad de la troza influye en la generación de residuos. Con respecto al aserrín, Otero (1985) indica que se genera en las tres fases principales del aserrio: aserrado, canteado y despuntado, y el volumen puede ser calculado matemáticamente con gran precisión. La Consulta Técnica de Tecnoforest (1982) afirma que el uso y manipuleo de residuos constituye una problemática que afecta sustancialmente los costos de producción. Ésta Consulta propone la producción de objetos pequeños para usar residuos de dimensiones reducidas. El Valor ornamental cubre el costo adicional de procesamiento y genera alguna utilidad. También propone el uso del aserrín para producción de tableros aglomerados de aserrín con mezclas de diferentes maderas. Este autor afirma que para cada densidad de tablero debe ensayarse una mezcla de aserrín adecuada.

\section{METODOLOGIA}

\section{INFORMACION PRELIMINAR Y ORGANIZACIÓN}

Se preparó un formato para la encuesta destinada a caracterizar las industrias de aserrío de Pucallpa. Se consideró aspectos generales, abastecimiento de materia prima, infraestructura, maquinaria y equipos, producción, productos 
elaborados y de recuperación, personal, capacitación, detección de problemas frecuentes y financiamiento.

Adicionalmente se recopiló información de la Dirección Regional Forestal y de Medio Ambiente sobre el número de aserraderos y producción en los años 1991 y 1992.

\section{TOMA DE DATOS}

La encuesta se llevó a cabo en 21 aserraderos, seleccionados de acuerdo a su ubicación: de la carretera Manantay de la carretera Federico Basadre y de la zona del río Ucayali (cuadro 1).

\section{TÉCNICAS DE MUESTREO}

Para determinar el tamaño de las muestras y el volumen por madera, se aplicó el muestreo estratificado al azar con distribución óptima y fijación proporcional.

\section{Cuadro 1}

\section{ASERRADEROS ENCUESTADOS}

\begin{tabular}{llc} 
ZONAS & \multicolumn{1}{c}{ ASERRADEROS } & TOTAL \\
\hline Zona río Ucayali & El Bosque, Tigrillo. Pacacocha, & \multirow{2}{*}{05} \\
Viena, Monteblanco & Maderera Manantay, Arbe, Unidos, & \\
Zona carretera & $\begin{array}{l}\text { Comapsa, Escurra, San Antonio, } \\
\text { Manantay }\end{array}$ & \\
& $\begin{array}{l}\text { Nueva Esperanza. Rocha, Vásquez, } \\
\text { Atalaya, Imecsa, Inforsa, Maria } \\
\text { Auxiliadora. }\end{array}$ & \\
Zona carretera & El Tambo. Torvisa, Inforesta, & 12 \\
Federico Basadre & Mapesa & 04 \\
\hline
\end{tabular}

\section{EVALUACION DE LAS ENCUESTAS}

La información recocida fue procesada, elaborándose cuadros de registros sobre la selección de opciones de los encuestados. 


\section{ELABORACIÓN DE FORMATOS PARA LA EVALUACIÓN DE RESIDUOS}

Se consideró los siguientes criterios:

a) Calificación de troza, en base a las especificaciones de la Guía de Cubicación Industrial propuesta por Fullop y Vásquez (1989).

b) Cuantificación de residuos generados en el proceso de aserrío discriminados como se especifica a continuación: aserrín, determinado por el producto del área de corte y espesor de la sierra; cantoneras, cantos y puntas, ara lo que se identificó en los aserraderos formas frecuentes y características de cada tipo de residuo; luego en laboratorio con réplicas a escala y mediante el método de determinación del volumen aparente por desplazamiento de agua, se establecieron factores de forma, que se aplicaban al cálculo del volumen de residuos a partir del máximo valor medido del espesor, ancho y longitud.

El cuadro 2 presente los aserraderos en donde se llevó a cabo la evaluación de residuos.

\section{Cuadro 2}

\section{ASERRADEROS DONDE SE EFECTUÓ LA EVALUACIÓN DE RESIDUOS}

\begin{tabular}{ll}
\hline MADERA & ASERRADEROS \\
\hline Catahua & Arbe, Manantay \\
Caoba & Vásquez, Unidos \\
Cedro & Manantay, Unidos \\
Copaiba & Nueva Esperanza \\
Cumala & Tigrillo, Rocha \\
Ishpingo & Manantay \\
Moena & Monte Blanco \\
Tornillo & COMAPSA \\
\hline
\end{tabular}




\section{RESULTADOS Y DISCUSION}

\section{CARACTERIZACIÓN DE LA INDUSTRIA DE ASERRÍO EN PUCALLPA}

\section{Abastecimiento de materia prima}

El volumen mensual promedio por aserradero es de 140000 pies tablares (330 m3). Cinco (24 \%) aserraderos declaran abastecerse exclusivamente por río, tres $(14 \%)$ lo hacen exclusivamente por la carretera Federico Basadre, y trece (26 $\%)$ se abastecen por ambos.

Con relación a la forma de abastecimiento, doce $(57 \%)$ aserraderos se abastecen mediante de extracción forestal propia, ocho (38 \%) compra madera rolliza a terceros y ocho (38\%) se abastecen por contratistas (habilitados). La frecuencia en el abastecimiento es como sigue: once (52 \%) aserraderos declaran tener materia prima durante todo el año, y ocho (38 \%) manifiesta que el abastecimiento es temporal: para madera de río, durante la época lluviosa, para madera de carretera, durante la época seca.

\section{Maderas procesadas}

Diecisiete (81 51) aserraderos encuestados procesan cedro, copaiba, caoba, tornillo, cumala, moena, catahua e Ishpingo. Cuatro (19\%) aserraderos procesan otras maderas como huayruro, shihuahuaco, cachimbo y pumaquiro.

\section{Fuente de energía}

Diecisiete $(81 \%)$ aserraderos cuentan con grupo electrógeno que genera energía eléctrica a partir del petróleo diesel $\mathrm{N}^{\mathrm{0}} 2$; tres $(14 \%)$ cuentan con grupo electrógeno y pueden abastecerse de la red pública; un $(5 \%)$ aserradero depende de la red pública.

\section{Capacidad instalada}

Dos $(10 \%)$ aserraderos tienen capacidad instalada menor a $10000 \mathrm{pt} /$ turno; seis $(29 \%)$ tienen una capacidad instalada de 12000 pt/turno; y tres (15\%) declaran procesar más de $15000 \mathrm{pt} /$ turno. Con excepción de un aserradero, todos los restantes observan el mismo horario de trabajo pudiendo establecerse comparaciones sobre producción. 


\section{Volumen de producción}

Como la producción de madera aserrada es discontinua, encuestando a ocho aserraderos que trabajan permanentemente, el volumen de producción promedio es de $180000 \mathrm{pt} / \mathrm{mes}$ y el consumo de madera rolliza promedio es de $242000 \mathrm{pt} / \mathrm{mes}$. De acuerdo a lo declarado por doce aserraderos (los nueve restantes no tenían información precisa), el rendimiento promedio de la materia prima procesada es de $73 \%$.

\section{Productos elaborados}

El principal producto de la actividad es la madera aserrada. Seis (29 \%) aserraderos se dedican exclusivamente a la producción de madera aserrada; trece (60 $\%)$ producen listones como producto de recuperación; seis (29 \%) producen palos de escoba; y seis $(29 \%)$ parquet. Ningún aserradero usa el aserrín y residuos de despuntado.

\section{Servicios ofrecidos}

Diecisiete $(81 \%)$ aserraderos prestan servicio de aserrío, tres $(14 \%)$ brindan servicio de reaserrío; cuatro (19 \%) dan servicio de moldurado; tres (14\%) dan servicio de secado; tres $(14 \%)$ da servicio de afilado; y uno $(5 \%)$ no prestan servicio alguno.

\section{Comercialización}

La comercialización de madera aserrada y productos elaborados a partir de residuos se efectúa en forma directa. Catorce $(66 \%)$ aserraderos venden sus productos sin la intervención de intermediarios o "corredores"; siete $(33 \%)$ declaran usar comisionistas. La producción se lotiza en función al peso y volumen, o a ambos, de una camionada, eventualmente, la madera se vende al por menor. Dieciocho $(86 \%)$ aserraderos destinan la producción al mercado de Lima y demanda local; cinco (24\%) exportan lotes variables de madera "rojas"; tres (14\%) envían la totalidad de la producción a sus propios depósitos en Lima.

\section{Dificultades en la industria}

Sobre las dificultades de las industrias de aserrío, se obtiene las siguientes opiniones: quince (71 \%) coinciden al afirmar que el abastecimiento de madera rolliza es el principal problema que afrontan actualmente; cinco $(24 \%)$ precisan que deben superar técnicas obsoletas; tres $(14 \%)$ sugieren la necesidad de planificar una 
organización más eficiente; siete $(33 \%)$ plantean la necesidad de contar permanentemente con mano de obra especializada; siete (33\%) opinan que la escasez de repuestos es un factor limitante para la producción; y tres (14 \%) afirman que existen otras dificultades prioritarias con financiamiento para operaciones y normas legales vigentes. Evidentemente, la práctica de técnicas obsoletas y la ausencia de mano de obra calificada influyen determinantemente en la calidad de la madera aserrada.

\section{Características del personal}

De los aserraderos encuestados se ha determinado un promedio de 27 trabajadores por planta; de jos cuales siete son capacitados. Siete (33 \%) aserraderos declaran usar ingenieros forestales en la extracción y peritos para apoyo técnico en planta. Diez (48 \%) declaran no requerir de asistencia técnica profesional. Cuatro (19\%) se abstuvieron de opinar.

\section{Capacitación}

Catorce (67 \%) aserraderos opinaron que requieren de cursos de capacitación para mantenimiento) de sierras de cinta; doce (57 \%) necesitan capacitación en mantenimiento de sierras circulares; seis (29\%) solicitan cursos sobre operaciones unitarias para el uso óptimo de materia prima; y siete (33 \%) desean cursos sobre cubicación; doce $(57 \%)$ en clasificación y control de calidad de madera aserrada; nueve (44\%) en gestión empresarial, siete (33\%) en operación y mantenimiento de maquinas de carpintería; dos (10\%) en mantenimiento de cuchillas para cortes periféricos; y tres $(14 \%)$ en otros aspectos.

\section{EVALUACION DE RESIDUOS}

El cuadro 3 presenta diámetros y longitud en promedio de las trozas, así como los valores promedio de volumen bruto, volumen de defectos y volumen total real. 


\section{Cuadro 3}

\section{DIMENSIONES DE LAS TROZAS}

\begin{tabular}{llcccrr}
\hline Madera & $\begin{array}{l}\mathrm{N}^{\circ} \text { de } \\
\text { troza }\end{array}$ & $\begin{array}{c}\text { Diámetro } \\
\text { promedio } \\
(\mathrm{m})\end{array}$ & $\begin{array}{c}\text { Longitud } \\
\text { promedio } \\
(\mathrm{m})\end{array}$ & $\begin{array}{c}\text { Volumen de } \\
\text { defectos } \\
\left(\mathrm{m}^{3}\right)\end{array}$ & $\begin{array}{c}\text { Volumen } \\
\text { total real } \\
\left(\mathrm{m}^{3}\right)\end{array}$ \\
\hline Catahua & 3 & 1,01 & 5,72 & 0,0156 & 0,53 & 15253 \\
Caoba & 5 & 0,90 & 4,25 & 0,0043 & 0,26 & 13,578 \\
Cedro & 7 & 0,70 & 3,92 & 0,0574 & 3,81 & 10,578 \\
Copaiba & 6 & 0,61 & 4,24 & 0,0367 & 2,81 & 7,040 \\
Cumala & 6 & 0,39 & 4,10 & 0,0002 & 0,03 & 3,1159 \\
Ishpingo & 7 & 0,58 & 3,68 & 0,0038 & 0,33 & 6,837 \\
Moena & 4 & 0,86 & 8,04 & 0,00 & 0,00 & 19,880 \\
Tornillo & 8 & 0,66 & 3,81 & 0,0461 & 2,56 & 10,191 \\
\hline
\end{tabular}

El diámetro real promedio de las trozas varía entre 0,39 para cumala y 1,01 $\mathrm{m}$ para catahua, el promedio general es de $0,71 \mathrm{~m}$. Comparando con los valores especificados por Tenazoa (1992) se advierte que el diámetro promedio de cumala está por debajo del mínimo establecido, otras están cerca del diámetro mínimo (copaiba, ishpingo y tornillo), circunstancia que influye en el rendimiento del aserrío. La longitud de trozas varían entre 3,92 para cedro y 8,04 m para moena, el promedio es de $4,72 \mathrm{~m}$. El volumen promedio de trozas varía entre 0,51 para cumala y $5,10 \mathrm{~m}$ para catahua, el promedio general es de $2,30 \mathrm{~m}^{3}$. El volumen de defectos evaluados según la metodología establecida por Fullop y Vásquez (1989) es cero para moena y 3,81\% para cedro; el promedio general es de 1,29\%. El volumen de defectos alcanza valores mínimos, por lo que en la evaluación de residuos no se considera esta variable. El volumen total real varía entre 3,059 para cumala y 19,880 $\mathrm{m}^{3}$ para moena; el promedio general es de $10,803 \mathrm{~m}^{3}$. El cuadro 4 presenta los valores porcentuales de aserrín y residuos sólidos. En la sierra principal, los valores promedio de aserrín, referidos al volumen total rollizo ingresado, varían entre 4,10 para caoba y $8,70 \%$ para cumala, el promedio general es de $6,04 \%$. Este valor relativamente alto en relación con el aserrín producido en la canteadora que es de $1.80 \%$ en promedio y en la despuntadora donde alcanza un valor promedio de 0.34 , se debe al espesor del elemento cortante, al área de corte y al pequeño diámetro de trozas, que al cuadrarlas producen mayor proporción de cantoneras, y aserradas producen tablas de 2 y 1 "generando mayor volumen de aserrín que si fueran de 3 o 4". En residuos sólidos, en la sierra principal se tiene promedio que oscilan entre 4,43 para copaiba y $12,30 \%$ para cumala, el promedio general es de 6,68 \%. En la canteadora se observa los mayores valores, el mínimo es de 15,67 para cedro y el máximo de 28,22 \% para moena, el promedio general es de 20,66\%. En la 
despuntadora se tiene valores que fluctúan entre 4,45 para moena y $15,67 \%$ para ishpingo, el Promedio general es de 10,23\%

\section{Cuadro 4}

\section{RESIDUOS DE ASERRÍO EN PORCENTAJE}

\begin{tabular}{|c|c|c|c|c|c|c|c|c|c|}
\hline \multirow{2}{*}{ Madera } & \multirow[b]{2}{*}{ Sierra } & \multicolumn{2}{|c|}{ Aserrín } & \multirow[b]{2}{*}{ Sierra } & \multicolumn{2}{|c|}{ Residuos Sólidos } & \multirow{2}{*}{$\begin{array}{l}\text { Total } \\
\text { Aserrín }\end{array}$} & \multirow{2}{*}{$\begin{array}{l}\text { Total } \\
\text { Sólidos }\end{array}$} & \multirow{2}{*}{$\begin{array}{c}\text { Total } \\
\text { General }\end{array}$} \\
\hline & & $\begin{array}{l}\text { Cantea } \\
\text { dora }\end{array}$ & $\begin{array}{l}\text { Despun- } \\
\text { teadora }\end{array}$ & & $\begin{array}{c}\text { Cantea- } \\
\text { dora }\end{array}$ & $\begin{array}{r}\text { Despun- } \\
\text { teadora }\end{array}$ & & & \\
\hline Catahua & 5,47 & 0.90 & 0.18 & 6,91 & 16.17 & 8.85 & 6.55 & 31.93 & 38.48 \\
\hline Caoba & 4.10 & 0,90 & 0.24 & 5,25 & 22,24 & 12.89 & 5.24 & 40.38 & 45,62 \\
\hline Cedro & 4.69 & 1,04 & 0,34 & 5,70 & 15.77 & 11,67 & 6.07 & 33,11 & 39.21 \\
\hline Copaiba & 6.57 & 3.65 & 0,31 & 4.43 & 20.29 & 8.51 & 10,53 & 33,23 & 44.06 \\
\hline Cumala & 8.70 & 4.00 & 0.13 & 12,20 & 23.05 & 9,15 & 12.83 & 44.40 & 57.23 \\
\hline Ishpingo & 6.20 & 1.66 & 0,64 & 5,34 & 18.74 & 15.67 & 8.50 & 39,75 & 48.25 \\
\hline Moena & 4.30 & 0,86 & 0,17 & 6,19 & 28.22 & 4,45 & 5.33 & 38,36 & 44.19 \\
\hline Tornillo & 8,32 & 1.69 & 0.67 & 7.41 & 20.80 & 10.67 & 10.68 & 38.88 & 49.56 \\
\hline
\end{tabular}

\section{CONCLUSIONES}

- Los principales residuos de aserrío de ocho maderas son: cantos (20,7 \%), despuntes $(10,2 \%)$, cantoneras $(6,7 \%)$, y aserrín $(8,2$

- Hay una relación entre diámetro de troza y rendimiento, que se observa en catahua y cumala.

- Los principales residuos factibles de ser industrializados son las puntas y el aserrín, ya que cantoneras y cantos tienen utilización.

\section{BIBLIOGRAFIA}

ACEVEDO, M. 1986. "Utilización de residuos y viruta de cepillado para fabricación de ladrillos de partículas". Laboratorio Nacional de Productos Forestales, Mérida Venezuela. 26 p.

CORNEJO, A. 1991. "Fomento de la producción, comercialización y asistencia técnica de la actividad forestal". Asociación de Extractores de Madera. Pucallpa. 
DIRECCION REGIONAL FORESTAL, FAUNA Y MEDIO AMBIENTE. 1991. Memoria Anual. Secretaría Regional de Asuntos Productivos Extractivos. Pucallpa.

FAO. 1980. "La industria de aserrío de maderas". Proyecto PNUD/FAO/PER/80/03. Lima. 16 p.

FULLOP y VASQUEZ, W. 1989. "Guía de cubicación industrial de maderas en trozas”. Proyecto de Desarrollo Industrial Forestal. Perú Canadá. Lima. 16 p.

GAUTHIER, D. 1986. "Diagnóstico de la industria de aserrío en Pucallpa". Programa de Desarrollo Industrial Forestal. Perú Canadá. Lima. 16 p.

OTERO, 1985. "Estudio sobre disponibilidad de sobrantes de madera para uso en la generación de energía eléctrica”. Electro Centro S.A. Pucallpa. 517 p.

TENAZOA, J. 1992. "Identificación de especies maderables". Primer Curso de Administración Forestal en la Región Ucayali. D.R.F.F. y M.A. Pucallpa. $95 \mathrm{p}$.

QUINTEROS, G. 1981. "Cuantificación de residuos en la industria del contrachapado a partir del capinurí”.

TECNOFOREST. 1982. Consulta Técnica. El libro de problemas. Feria Internacional del Pacífico. Lima. 56 p. 